\title{
Wind Farm Dynamic Equivalence Based on the Wind Turbine Output Active Power Sequence Clustering
}

\author{
Zhang Ge, Yang Yiyun and Xiao Jing \\ Guangxi Power Grid Company Electric Power Research Institute, Nanning, 530000
}

\begin{abstract}
In order to reduce the complexity of simulation model containing wind farms in the context of keeping the accuracy static, this paper put forward a kind of Dynamic Equivalence method aiming at making output characteristic of the connecting point of wind farm consistent. Based on the output power sequence of wind turbines, geometric template matching algorithm is used to obtain the characteristic of that power sequence and then Attribute Threshold Clustering Algorithm is used to classify wind turbine. In each cluster, the parameter of wind turbine is made equal according to the principle of constant power output character and then be distinguished according to AMPSO. At last, this paper takes a practical wind farm as an example and respectively simulates the conditions of fault of system side and variation of wind speed, which is used in comparing the output characteristic of detailed model and Equivalent model. Results show that the output characteristic of the connecting point of wind farm keeps consistent after equivalent and that the Clustering Algorithm can reflect the operating characteristics of the wind turbine in the whole moment of any time period. It can also be saw that Equivalent method is reasonable and effective, which has certain value in engineering application.
\end{abstract}

\section{Introduction}

In the simulation of large wind farm connecting into power grid, it is a heavy work if we modelling every wind turbine, which will also affect the speed, accuracy and convergence of algorithm and thus it is necessary to make Dynamic Equivalent model of wind farm. Currently, Dynamic Equivalent method of wind farm mainly includes single-generator equivalent method, semi equivalent method and multi-generator equivalent method [1], [2]. Among the three methods, singlegenerator equivalent method is simple but has the lowest accuracy and multi-generator equivalent method has the highest accuracy but is complicated while semi equivalent method behaves in between of two methods.

Multi-machine equivalent firstly need to divide wind turbines into a plurality of clusters and then make equivalence of each clusters, among which it is essential to solve how to realize the equivalence of each clusters [3]-[6]. Reference [7] classifies the wind turbine with the standard of transient track of terminal voltage but its adaptability is not wide for there is less condition of fault in actual operation. Moreover, reference [8], [9] put forward a feasible classification method of time data from wind turbine that classifying time sequence of measured active and reactive power by spectral clustering algorithm based on diffusion mapping theory.

This paper takes the output active power of wind farm as time sequence and put forward a new classification method based on geometric template matching and attribute threshold clustering algorithm, and then distinguish the parameter of wind turbine by AMPSO. At last, based on DIgSILENT/ Power Factory, the accuracy and adaptability of the equivalent model is verified.

\section{The wind turbine clustering algorithm}

\subsection{Similarity algorithm for geometric template matching}

The geometric sample matching algorithm can take into account the dynamic change process of the system, and the calculation efficiency is higher [10], [11]. Firstly, the time delay embedding method is used to analyze the phase space reconstruction of active power sequence, and then to analyze the dynamic characteristics of the wind turbine, and then evaluate the difference of each reconstruction vector with the data window and similarity concept. Finally, the similarity is transformed into the distance matrix of the sample, and it provides the basis for the subsequent clustering algorithm.

By meaning time delay, the phase space reconstruction can be reconstructed into a dimensional space vector at different times and the chaotic behavior of the system is studied. Assuming a single variable of time series is $x(t)(t=1,2, \ldots, n)$. After the time delay, the new $m$ dimension vector $X(t)$ can be obtained: 


$$
\begin{aligned}
& X(t)=\{x(t), x(t+\tau), \ldots, x(t+(m-1) \tau)\} \\
& t=1,2, \ldots, M, M=n-(m-1) \tau
\end{aligned}
$$

In the formula: $\tau$ is the time delay; $m$ is the embedding dimension; $M$ is the phase point number.

The embedding dimension $\mathrm{m}$ and time delay $\tau$ is given as follows: given embedding dimension $\mathrm{m}$, the time delay is increased, and the complex auto correlation function $\mathrm{R}(\tau)$ is calculated as:

$$
R(\tau)=\frac{1}{M} \sum_{i=1}^{M} \sum_{j=1}^{m-1}\{[f(x(i))-f(\bar{x})] \cdot[f(x(i+j \tau))-f(\bar{x})]\}
$$

$$
f(x)=1+x+x^{2}+\ldots+x^{m-1}
$$

In the formula: $\bar{x}=\frac{1}{n} \sum_{t=1}^{n} x(t)$;

Geometric sample matching algorithm can be used to compare the similarity of two time series. For time series $\mathrm{x}_{1}(\mathrm{t})$ and $\mathrm{x}_{2}(\mathrm{t})(\mathrm{t}=1,2 \ldots \mathrm{n})$, the same time delay $\tau$ and embedding dimension $\mathrm{m}$ are used to reconstruct the phase space. Two new $\mathrm{m}$ dimensional vector $\mathrm{X}_{1}(\mathrm{t})$ and $\mathrm{X}_{2}(\mathrm{t})$ $(\mathrm{t}=1,2, \ldots, \mathrm{M})$ can be obtained. Nearest neighbor elements of $\mathrm{K}$ in vector $\mathrm{X}_{1}(\mathrm{t})$, which is the nearest to the distance vector $\mathrm{X}_{2}(\mathrm{i}), X_{1}\left(n_{i, 1}\right), \ldots X_{1}\left(n_{i, \mathrm{k}}\right)$, The expression of $n_{i, k}$ is:

$$
\left\{\begin{array}{l}
n_{i, 1}=\underset{j}{\arg \min }\left\|X_{2}(i)-X_{1}(j)\right\| \\
\cdots \\
n_{i, \mathrm{k}}=\underset{j \neq n_{i, 1}, \ldots, n_{i, 1}}{\arg \min }\left\|X_{2}(i)-X_{1}(j)\right\|
\end{array}\right.
$$

In the formula, function $\underset{x}{\arg \min } f(x)$ is to find the $\mathrm{x}$ so that the value of $\mathrm{F}(\mathrm{x})$ is the smallest.

The similarity of vector $X_{1}(t)$ and vector $X_{2}(t)$ can be expressed as:

$$
S\left(X_{1}, X_{2}\right)=\frac{1}{M-1} \times \sum_{i=1}^{M-1} \frac{\left[\bar{X}_{1}(i+1)-\bar{X}_{1}(i)\right] \cdot\left[X_{2}(i+1)-X_{2}(i)\right]}{\left\{\max \left[\left|\bar{X}_{1}(i+1)-\bar{X}_{1}(i)\right|,\left|X_{2}(i+1)-X_{2}(i)\right|\right]\right\}^{2}}
$$

In order to use the distance to replace the similarity, definition:

$$
d\left(X_{1}, X_{2}\right)=\exp \left(-S\left(X_{1}, X_{2}\right)\right)
$$

\subsection{Characteristic threshold clustering algorithm}

Characteristic threshold clustering algorithm does not need to specify the number of $\mathrm{K}$ in advance [12], and for the same sample, it is the same every time the running results. In fact, the steps are as follows:

1) Calculate the distance between the two samples.

2) Given the classification of clustering radius d.

3) Set up all samples candidate classes, and make candidate class contain samples within radius d.

4) If the samples in a candidate class are maximum, then it is the first class, and will be removed.
5) Repeat steps 3 and 4 until the remainder of the sample is classified into a certain class.

Based on the cluster method described above, wind farm cluster can be obtained, as shown in Figure 1.

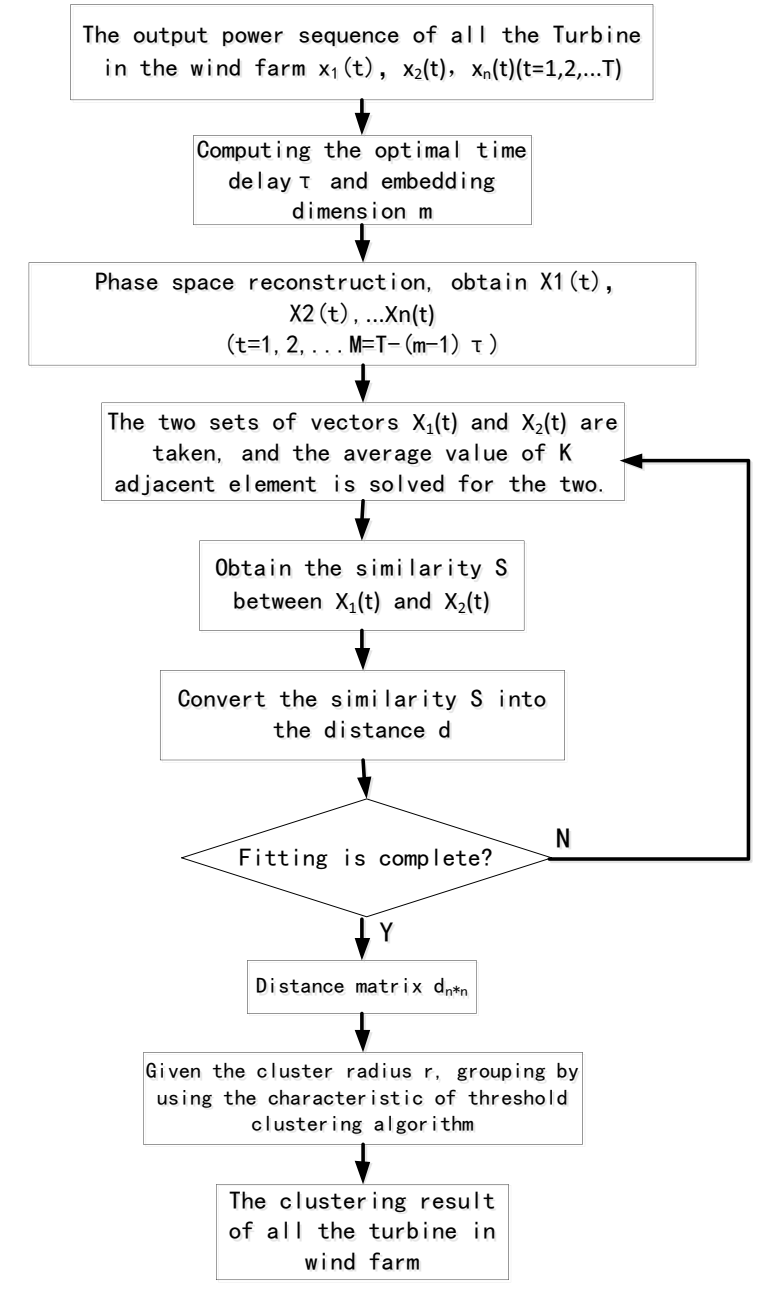

Figure 1. The classification flow chart of wind farm.

\section{PMSG parameter identification based on AMPSO}

In view of the close correlation of the output active power and its dynamic characteristics of PMSG, this paper chooses the square sum of the output active power of the wind farm before and after the equivalent function to find the optimal parameters of the PMSG. The specific implementation steps of PMSG parameter identification based on AMPSO are as follows:

1) Get system input data including $u_{d}, u_{q}, i_{d}, i_{q}$, $\omega_{m}$, these five variables are measured by the actual motor, so as to ensure that the algorithm is feasible in practical application. In order to improve the accuracy of parameter identification, it is necessary to sample enough samples; this paper intends to sample 1800 sets of data.

2) The position and velocity of the particles are initialized, the size of the particle population, the number of iterations, the time step, the convergence speed, the random attenuation factor, and the upper and lower limits of the parameters $L_{d}, L_{q}, R_{s}, \psi_{f}$ are set up. 
3) To calculate the fitness value of particles, compare the fitness value of individual particles and individual extreme value, update the existing individual extreme value, and compare the fitness value of individual particle and global extreme value, and update the existing global extreme value.

4) To update the speed and position of particles, and to carry out mutation operation of $2 \%$ particles, thus forming a new particle swarm. Among them, the first iteration, the particle velocity and position update rules are as follows:

$$
\begin{aligned}
& v_{i k}(n+1)=w v_{i k}(n)+c_{1} \times \operatorname{rand} 1(0,1) \times\left(p_{i k}(n)-x_{i k}(n)\right)+ \\
& c_{2} \times \operatorname{rand} 2(0,1) \times\left(p_{g k}(n)-x_{i k}(n)\right) \\
& x_{i k}(t+1)=x_{i k}(t)+v_{i k}(t+1)
\end{aligned}
$$

$\omega$ is the inertia weight coefficient; $c_{1}, c_{2}$ are the learning factors; the rand $1(0,1)$ and rand $2(0,1)$ are two random numbers of $(0,1)$.

5) Repeat steps 3 and 4 to calculate the fitness value of each particle, if the fitness value of a certain iteration is less than the threshold set in advance, then stop searching, the solution vector is the optimal parameter of PMSG.

\section{Numerical simulation}

\subsection{Division of wind power plants}

Select the active power sequence of 24 typhoon machine output in Figure $3 x_{1}(t), x_{2}(t), \ldots x_{24}(t)(\mathrm{t}=1,2, \ldots$, 1501). The phase space reconstruction of 24 active power series is carried out, so we got the reconstruction vector $X_{1}(t), X_{2}(t), \ldots X_{24}(t)(\mathrm{t}=1,2, \ldots, 1501)$, optimal embedding dimension $=4$, time delay $\tau=11$. To reconstruct the vector as the input, solve adjacent element number $\mathrm{k}=5$ between any two sets of vector, a matrix $n_{M \times k}$ was obtained between every two groups; the similarity $S_{24 \times 24}$ between two sets of vectors is obtained; converted the similarity $\mathrm{S}$ to the distance $\mathrm{d}$, so got the distance matrix $d_{24 \times 24}$; Finally, clustering algorithm is used to classify the clusters based on attribute threshold clustering algorithm, set clustering radius $r_{0}=0.5$, the clustering result is shown in Figure 2.

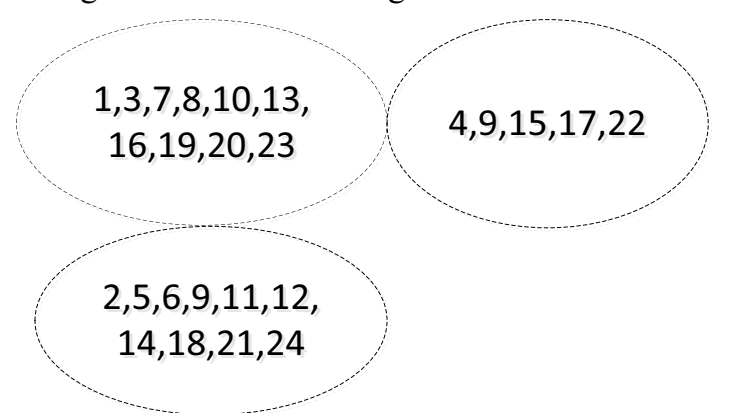

Figure 2. The clustering result of wind turbine.

\subsection{Equivalent model parameter identification}

Set the maximum number of iterations for the 200 time, adaptive mutation probability is $0.002 \%$, the fitness curves of AMPSO are shown in Figure 3.

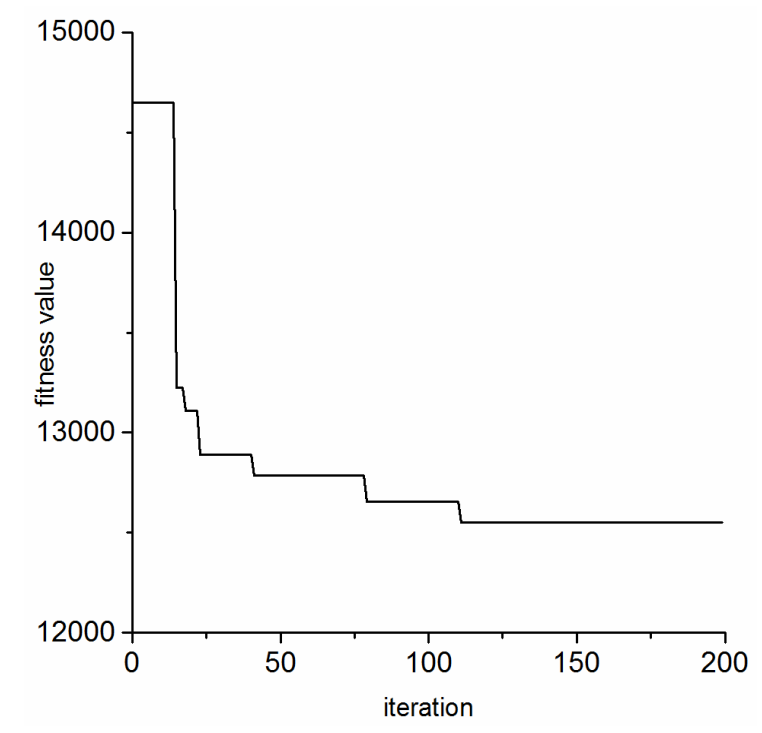

Figure 3. The AMPSO fitness curve.

By using AMPSO identify PMSG, the optimal parameters of the wind turbine can be obtained: $L_{d}=L_{q}=1.432 \mathrm{mH}, R_{s}=0.00082 \Omega, \psi_{f}=6.774 \mathrm{~Wb}$.

\subsection{Dynamic simulation}

In order to verify the correctness of the proposed method in this paper, we use DIgSILENT/Power Factory to simulate, the active power and reactive power are shown in Figure 4-6.

1) Gust, start at $2 \mathrm{~s}$, stop at $8 \mathrm{~s}$, maximum wind speed is $3 \mathrm{~m} / \mathrm{s}$.

2) Wind speed increase, start at $2 \mathrm{~s}$, stop at $8 \mathrm{~s}$, maximum wind speed is $4 \mathrm{~m} / \mathrm{s}$.

3) Three-phase short-circuit fault occurred at grid side, the failure occurs at $8.2 \mathrm{~s}$, disappeared at $8.2 \mathrm{~s}$.

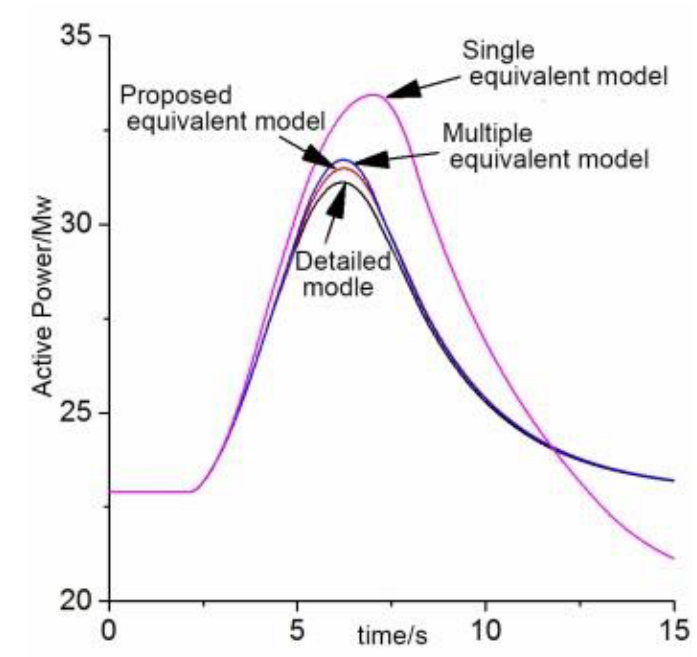

(a) Active power change curve 


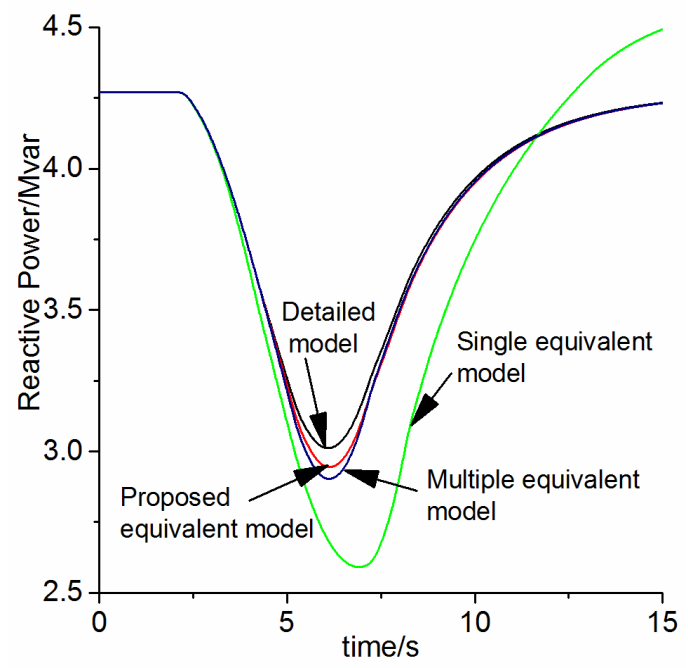

(b) Reactive power change curve

Figure 4. The Output curve of wind power plants in gust.

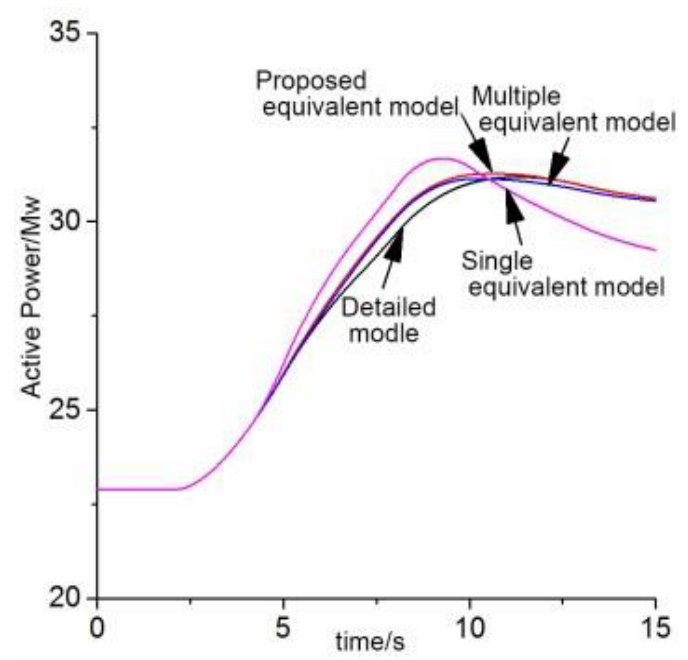

(a) Active power change curve

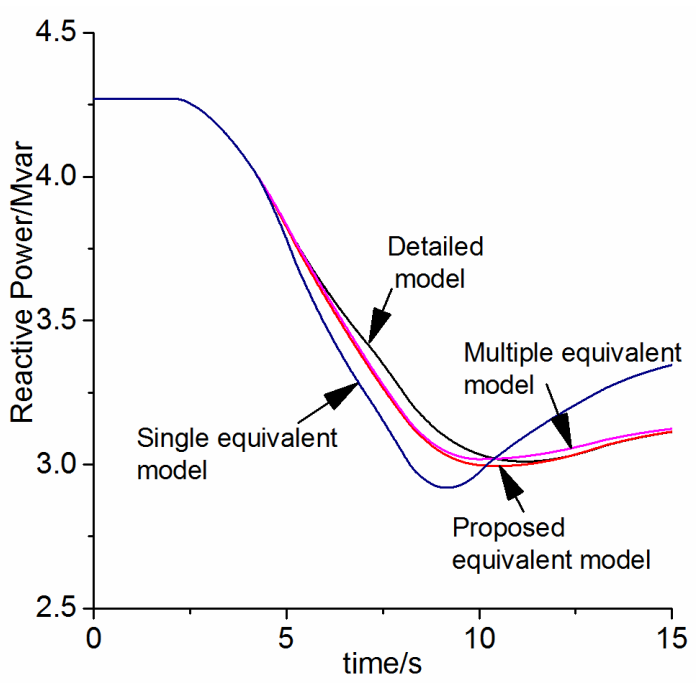

(b) Reactive power change curve

Figure 5. The Output curve when wind speed increasing.

It can be seen in Figure 4, Figure 5 and Figure 6 the equivalent effect on the output characteristics of the equivalent model and the detailed wind power plants model is of high degree coincidence; compared with the equivalent model of single well, the multi machine equivalent model is better, which also explained the necessity of wind power generators. From the view of adaptability, under the gradient wind and gust disturbance, the equivalent model can response and maintain the consistency of the detailed model with, this also proves that all the time in the equivalent method of clustering result can be applied to the period of time.

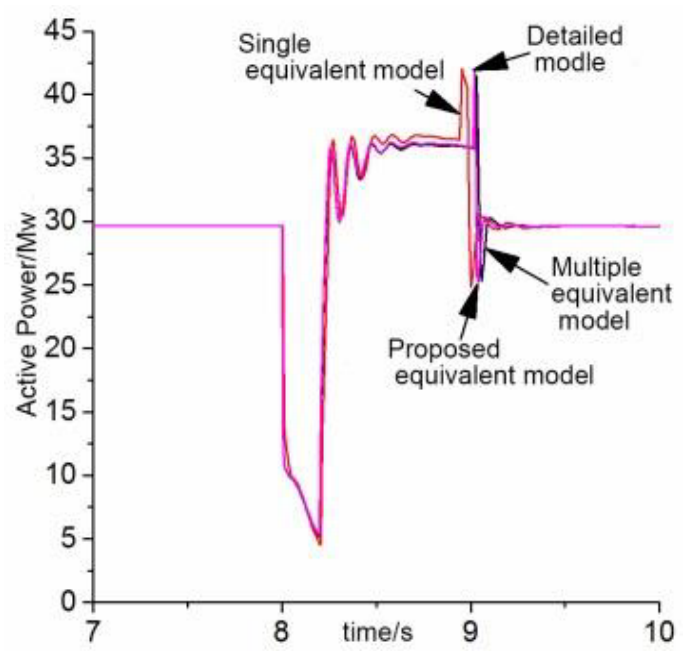

(a) Active power change curve

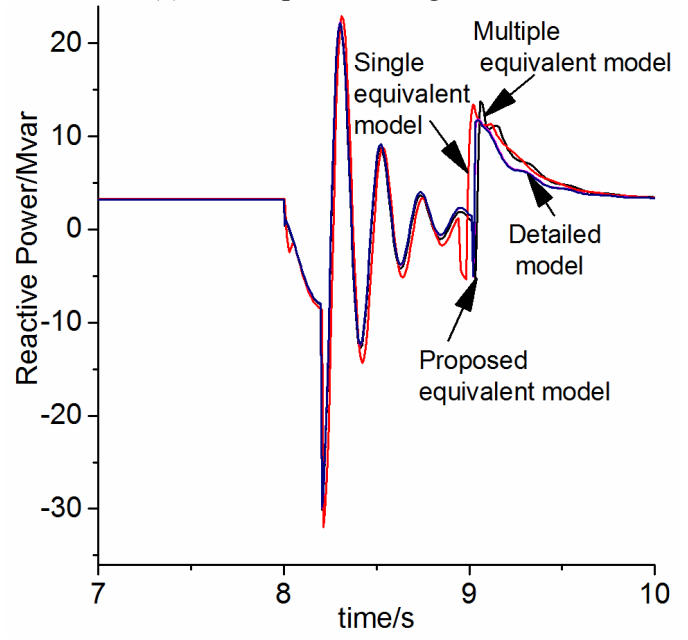

(b) Reactive power change curve

Figure 6. The wind power plants output curve of the system side fault.

\section{Conclusions}

The wind power plants dynamic equivalence method is proposed in this paper to the time series data of the power output of the wind turbine for clustering the sample data, using the geometric template matching and attribute threshold clustering algorithm for clustering, the clustering method can reflect the operating characteristics of wind turbine, and the clustering result is suitable for all the time during the period of time, can avoid wind turbine multiple groups.

The output characteristics of the wind power plants in three different conditions are compared with the simulation results in DIgSILENT/ Power Factory. The results show that the dynamic equivalent method of the wind power plants proposed in this paper can reflect the dynamic characteristics of the wind power plants. 


\section{References}

1. M. A. Chowdhury, W. X. Shen, N. Hosseinzadeh, et al., A novel aggregated DFIG wind farm model using mechanical torque compensating factor, Energy Conversion and Management, (67):265-274, (2013)

2. H. Z. Liu, Z. Chen, Aggregated modelling for wind farms for power system transient stability studies, 2012 Asia-Pacific Power and Energy Engineering Conference (APPEEC), Shanghai, China: IEEE, (2012):1-6

3. V. Akhmatov, H. Knudsen, An aggregate model of a grid-connected, large-scale, offshore wind farm for power stability investigations importance of windmill mechanical system, Electrical Power Energy Systems, 24(9):709-717, (2002)

4. Z. Q. Mi, X. W. Su, Y. Yang, et al., Study on dynamic equivalent model of wind farms with wind turbine driven doubly fed induction generator, Automation of Electric Power Systems (in Chinese), 34(17):72-77, (2010)

5. S. Y. Chen, C. Wang, H. Shen, et al., Dynamic equivalence for wind farms based on clustering algorithm, Proceedings of the CSEE (in Chinese), 32(4):11-19, (2012)

6. Y. Q. XU, N. Wang, Study on dynamic equivalence of wind farms with DFIG based on clustering analysis, Journal of North China Electric Power University (in Chinese), 40(3):1-5(2013)

7. Z. Q. Mi, X. W. Su, Q. X. Yang, et al., Mutimachine representation method for dynamic equivalent model of wind farms, Transaction of China Electrotechnical Society (in Chinese), 25(5):162-169, (2010)

8. Y. Ma, T. Runolfsson, J. N. Jiang, Cluster analysis of wind turbines of large wind farm with diffusion distance method, IET Renewable Power Generation, 5(2):109-116, (2011)

9. L. Li, Y. Chen, Wind turbine grouping with spectral clustering algorithm based on diffusion mapping theory, Electric Power Automation Equipment (in Chinese), 33(6): 113-118, (2013)

10. F. Jordan, M. Shie, P. Joelle, et al., Time series analysis using geometric template matching, IEEE Transactions on Pattern Analysis and Machine Intelligence, 35(3):740-754, (2013)

11. $\mathrm{Xu}$ rock, the estimation of the parameters of the phase space reconstruction of chaotic time series, Chongqing: Chongqing School, (2013)

12. J. H. Laurie, K. Semyon, Y. Shibu, Exploring expression data: Identification and analysis of coexpressed genes, Genome Research, 9:1106-1115, (1999) 